\title{
UTILIZACIÓN CONJUNTA DE LA ENCUESTA PRESENCIAL Y TELEFÓNICA EN LAS ENCUESTAS ELECTORALES
}

\author{
JOINT USE OF FACE-TO-FACE AND TELEPHONE \\ SURVEYS IN ELECTORAL SURVEYS
}

\author{
VIDAL DíaZ de RADA vidal@unavarra.es \\ Departamento de Sociología, Universidad Pública de Navarra. España
}

\begin{abstract}
Resumen
Numerosos investigadores sostienen que la encuesta telefónica realiza estimaciones de voto más precisas que la encuesta personal. A estas alturas nadie duda de las ventajas de la encuesta telefónica en cuanto a la representatividad, calidad de la información recogida, mayor rapidez y menor coste; si bien la encuesta telefónica presenta también importantes inconvenientes. Debemos considerar también la presencia de nuevas barreras que dificultan la accesibilidad (por ejemplo contestador automático); y saturación del medio telefónico por la gran utilización en actividades de publicidad y telemarketing que genera un gran número de llamadas "infructuosas" y entrevistas interrumpidas. El objetivo del presente trabajo es valorar la adecuación de las encuestas telefónicas en la predicción electoral, tratando de detectar si presentan mejoras sustantivas respecto a las encuestas presenciales.
\end{abstract}

\section{Palabras Clave}

Calidad de la encuesta, Encuestas mixtas, Procedimientos mixtos de encuesta, Tasa de respuesta, Sesgo de no respuesta.

\section{Abstract}

Several researchers declared that the telephone survey reaches more accurate voting tendencies than the face-to-face surveys. Telephone survey shows numerous advantages compared to a face-to-face one but, however, the telephone survey also has some inconveniences. There are also some new barriers which make access more difficult (e.g. the automatic answering phone) and the saturation of the telephone medium because of the large amount of publicity activities which generate a large number of "unsuccessful" calls and interrupted interviews. The objective of this paper is evaluating the adaptation of the telephone surveys in the electoral forecasts; in an attempt to see if it shows substantial improvements when they are compared to face-to-face interviews.

\section{KEYWORDS}

Data Quality, Mixedsurvey, Mixed Mode Data Collection, Non-Response Bias, Response Rates, Telephone Survey. 


\section{VentajAs E INCONVENIENTES de LA ENCUeSTA TELEFóNICA}

A estas alturas nadie duda de las ventajas de la encuesta telefónica en cuanto a la representatividad, la calidad de la información recogida, la mayor rapidez y el menor coste (DeLeeuw 2008:133-135). Respecto a la "representatividad", la encuesta telefónica proporciona una fácil accesibilidad a la población objeto de estudio; facilidad para realizar más de un intento para contactar con un hogar; sencillez para detectar las unidades muestrales no pertenecientes al universo; mayor aleatoriedad en la realización de "sustituciones" (empleo de "reservas"); mayor dispersión muestral sin incremento del coste; $y$ facilidad para contactar con determinados estratos sociales que presentan problemas de accesibilidad. Respecto a la calidad de la información recogida, produce una mayor sensación de anonimato que suele redundar en un aumento de sinceridad en las respuestas ${ }^{1}$; menor influencia del entrevistador; facilidad para controlar al entrevistador y detectar errores con rapidez; y posibilidad de eliminación de la contaminación entre preguntas. Todo esto genera una gran "rapidez" en la recogida de información y en el análisis de los resultados, bajo coste y una mayor facilidad para adaptarse a los horarios del universo objeto de estudio.

No obstante, y pese a lo señalado en el párrafo anterior, presenta también inconvenientes relacionados con la escasa calidad de los marcos muestrales, baja tasa de respuesta de determinados colectivos²; presencia de "nuevas barreras" que dificultan la accesibilidad (por ejemplo, el contestador automático) y "saturación" del medio telefónico por la gran utilización en actividades de publicidad y telemarketing que genera un gran número de llamadas "infructuosas" y entrevistas "interrumpidas" (Gwartney 2007:72-73). A estos hay que añadir los derivados de la falta de "cobertura", bien por la ausencia de teléfono en algunos hogares ${ }^{3}$ y por la gran expansión de la telefonía móvil, que está sustituyendo a la telefonía fija. Respecto a esta última, hay que señalar que a finales del año 2009 el 19\% de la población española dispone únicamente de teléfono móvil; porcentaje que aumenta notablemente en los sectores de menor edad (Instituto Nacional de Estadística 2010).

No hay acuerdo en los investigadores españoles sobre la mejor adecuación de la encuesta presencial o telefónica para la investigación electoral, entre otras razones por la falta de investigaciones sobre el tema en España, caso muy distinto de lo que sucede en otros países (entre otros, Keeter et al., 2000; Nicolaas et al., 2000; Wessel et al., 2000; Green et al., 2001; Holbrook et al., 2003; Ellis y Krosnick 1999; Voogt y Saris 2005;

${ }^{1}$ Hay investigadores que señalan el efecto contrario, por ejemplo: Krosnick (1991; 1999), Green et al. (2001); Holbrook et al. (2003).

${ }^{2}$ Personas de más edad, bajos niveles de estudios, etc.

${ }^{3}$ Los hogares sin teléfono se caracterizan por tener menos recursos económicos, menores niveles educativos, más edad, etc. Se trata, concretamente, de los colectivos más vulnerables socialmente; tal y como han señalado Green et al. (2001:2). 
Jäckle et al., 2006). Que nos conste, en España existe únicamente un trabajo sobre el tema (Martínez de Luna 2008), y se trata de una investigación donde se da cuenta de importantes diferencias entre la estimación realizada por las encuestas personales y las telefónicas: en la página 16 se señala que "las encuestas telefónicas presentan una mayor tasa de no respuesta parcial ('no sabe' y 'no contesta') tanto en el recuerdo de voto como en la intención de voto" (Martínez de Luna 2008:16). Respecto al recuerdo de voto, y "considerando las desviaciones voto con respecto a los resultados electorales reales, las opciones electorales que mediante encuesta personal aparecen sobrerrepresentadas habitualmente -así como las fuerzas que resultan infrarrepresentadas- agudizan esas tendencias en las encuestas telefónicas"; lo que implica que éstas (encuestas telefónicas) producen un mayor desajuste (Martínez de Luna 2008: 16).

El presente trabajo continúa en esta línea interpretativa con el fin de valorar la adecuación de las encuestas telefónicas en la predicción electoral, tratando de detectar si presentan mejoras "sustantivas" respecto a las encuestas presenciales. Para ello se utilizará una investigación realizada por el Centro de Investigaciones Sociológicas en las últimas elecciones autonómicas de Galicia (19 de junio del año 2005) que utilizó dos muestras similares: una realizada con una encuesta presencial y otra con una encuesta telefónica. El objetivo es comparar los resultados localizados por ambas con el fin de responder a las siguiente hipótesis:

H1: La tasa de rechazo a responder el cuestionario y de abandonos una vez comenzado son similares en ambos procedimientos (Martínez de Luna 2008:15); en contra de lo hallado en otros contextos donde la encuesta presencial genera una mayor tasa de rechazo y una menor tasa de abandonos una vez comenzada la entrevista (Groves 1989:139; Ellis et al. 2005:11).

H2: Existen importantes diferencias muestrales entre ambos procedimientos, diferencias que pueden afectar notablemente a la predicción electoral. Diversas investigaciones han señalado que las muestras obtenidas con encuestas telefónicas presentan un mayor nivel educativo, más ingresos y menor edad media de los entrevistados.

H3: Se aprecian también notables diferencias en la "calidad en la respuesta" como consecuencia del procedimiento de recogida de información; "calidad en la respuesta" definida como número de preguntas no respondidas ('no sabe' y 'no contesta') y en la presencia de determinados efectos en la respuesta como son el sesgo de "deseabilidad social", obtención de respuestas más extremas, etc. De hecho, uno de los objetivos de la realización de este trabajo es analizar hasta qué punto el procedimiento de recogida de información presenta influencia sobre una temática "sensible" o "delicada" como es la intención del voto político (Méndez y Martínez 2007:4).

H4: En cuanto a la estimación del voto, las encuestas telefónicas producen una mayor respuesta de la intención del voto futuro (esto es, menos abstención). También generan unas respuestas más sinceras (un mejor acercamiento al resultado electoral), según se desprende al comparar los resultados con otras investigaciones y con los resultados electorales. 
Es importante precisar que este trabajo no considera la adecuación o ajuste del modelo de estimación utilizada, ni tampoco el cambio que ha podido producirse en el electorado entre el momento en el que respondió la encuesta y el día de las elecciones. Son aspectos que quedan fuera del objetivo de este trabajo.

\section{INVESTIGACIÓN PREELECTORAL SOBRE LAS ELECCIONES AUTONÓMICAS DE 2005: DeTALLES TÉCNICOS}

Con el fin de comprobar las hipótesis propuestas utilizaremos el estudio preelectoral de Galicia para las elecciones autonómicas del 19 de junio del año 2005 realizado por el Centro de Investigaciones Sociológicas (estudio número 2.608). El trabajo de campo se realizó del 26 de mayo al 5 de junio de 2005 utilizando conjuntamente dos procedimientos de recogida de información: encuestas personales o presenciales y encuestas telefónicas con el sistema CATI; realizando 1600 entrevistas con cada procedimiento. Todas se realizaron en las viviendas de los entrevistados: en las entrevistas presenciales las viviendas fueron seleccionadas por medio de rutas aleatorias, y en las telefónicas se realizó una selección aleatoria a partir de la base de datos de los teléfonos de los municipios. Las personas entrevistadas (unidades últimas) han sido seleccionadas utilizando cuotas de sexo y edad (en el Anexo se presentan los detalles técnicos de la investigación).

Para facilitar la comparabilidad entre los procedimientos se buscó la mayor similitud entre ambos utilizando una selección muestral similar, los mismos municipios como puntos de muestreo, un único cuestionario, mismas fechas para el trabajo de campo, etc., siguiendo así las recomendaciones de Bowers y Ensley (2003:1-2). de con el fin que la investigación cumpliera las "ocho características necesarias para conocer los efectos del procedimiento de investigación", tal y como han sido propuestas por Holbrook et al. (2003:88).

Las 3.205 encuestas realizadas presentan un error muestral del $\pm 1,8 \%$ para el conjunto de la muestra, considerando un nivel de confianza del 95,5\% (dos sigmas), y $P=Q$. Se ha utilizado un muestreo polietápico, estratificado por conglomerados, con selección de las unidades primarias de muestreo (municipios) y de las unidades secundarias (secciones) de forma aleatoria proporcional. Los estratos se han formado por el cruce de la provincia con el tamaño del hábitat, dividido en 5 categorías: menos 0 igual a 2000 habitantes; de 2001 a 10.000; de 10.001 a 50.000; de 50.001 a 100.000, y de más de 100.000 .

\section{RESPUESTA CONSEGUIDA: NÚMERO DE RESPUESTAS Y REPRESENTATIVIDAD}

En este apartado se analiza la "calidad" de la respuesta conseguida, calidad definida considerando la "similitud con el universo"; esto es, ausencia de diferencia entre la población objetivo y la población medida. La calidad será mayor en la medida que se consiga la máxima "cobertura" y una "elegibilidad" total. La cobertura se refiere a 
los elementos del universo (población objetivo) que no son incluidos en el marco poblacional; esto es, aquellos elementos que no pueden ser localizados al no formar parte del marco utilizado ${ }^{4}$. La "elegibilidad", por su parte, hace referencia a la probabilidad (equiprobabilidad) de seleccionar a todos los elementos del universo.

Con el fin de conocer con precisión el número de contactos efectuados para realizar cada cuestionario utilizaremos la "información sobre el trabajo de campo" que se recoge en la última hoja de los cuestionarios del CIS: incidencias en la selección del entrevistado, momento en que se ha realizado la entrevista (fecha, día de la semana, duración y hora) y valoración del entrevistador sobre el desarrollo de la entrevista y la sinceridad del entrevistado. De toda esta información centraremos la atención en las incidencias en el proceso de localización del encuestado, definidas como los "acontecimientos" que tienen lugar entre la planificación de la recogida de información y lo que realmente se obtiene; la diferencia entre lo planificado y lo obtenido (Díaz de Rada 2006:3).

La ficha de incidencias utilizada por el CIS se muestra en el Cuadro 1 y para completarla el encuestador debe anotar el "número de veces" que ha ocurrido cada una de estas situaciones hasta efectuar la entrevista. Es decir que cada cuestionario lleva unido, junto a la información propia de su temática, los problemas que han tenido lugar para localizar cada entrevistado. Las incidencias se recogen en variables cuyos valores oscilan entre el 0 y el 99 , de modo que es posible que un entrevistador no encuentre ninguna incidencia (esto es, que realice una entrevista al establecer el primer contacto con la vivienda), aunque también puede contabilizar 99 incidencias (e incluso más) antes de conseguir la entrevista.

\section{Cuadro 1.}

Tabla de incidencias utilizada por el Centro de Investigaciones Sociológicas.

ENTREVISTADOR: anotar el "número de veces" que tiene lugar cada una de las siguientes situaciones antes de efectuar una entrevista

I.2 Dificultad de acceso al edificio, casa, urbanización, etc.

I.3 Viviendas en las que no hay nadie

I.4 Viviendas en las que se niegan a recibir ninguna explicación

I.5 Negativas de varones a realizar la entrevista

I.6 Negativas de mujeres a realizar la entrevista

I.7 Contactos fallidos por no cumplir cuotas

I.8 Contactos fallidos por no ser una vivienda (oficinas, consultas, etc.)

Fuente: Centro de Investigaciones Sociológicas (2005a).

\footnotetext{
${ }^{4}$ Por ejemplo, las personas que disponen únicamente de teléfonos móviles, al no disponer de directorios telefónicos de estos números, no pueden ser incluidos con el marco muestral.
} 
En la parte derecha de la Tabla se presenta la distribución porcentual de las incidencias, donde puede apreciarse el elevado número de contactos a "no hogares" (44\%), a viviendas en las que no hay nadie $(26 \%)$ y a viviendas donde no se ha podido llevar a cabo la entrevista por estar cubiertas las cuotas de las personas seleccionadas (15\%). Debe tenerse en cuenta que estas tres razones son responsables del $85 \%(44+26+15)$ de la totalidad de las incidencias cometidas. Ahora bien, los "contactos fallidos por no ser una vivienda" (no hogares) no constituye, en sí mismo, una incidencia, sino más bien se trata de "fallos en el marco muestral"; por lo que deberían ser eliminados del cálculo de incidencias (Fowler 2002:40). Eliminar esta razón supone un descenso de las incidencias a 14.179; que implica que para conseguir una entrevista deba contactarse con diez personas. Esta "readaptación" de los porcentajes genera que las razones que producen más incidencias sean el contacto a viviendas vacías (casi la mitad de las llamadas infructuosas se producen por este motivo) y los contactos a hogares con cuotas cubiertas (uno de cada cuatro).

Tabla 1.

Incidencias en el proceso de localización del entrevistado en las entrevistas presenciales. Distribución porcentual de las incidencias.

\begin{tabular}{lrc}
\hline & $\begin{array}{c}\text { Con todas } \\
\text { las incidencias }\end{array}$ & $\begin{array}{c}\text { Sin "no } \\
\text { viviendas" } \\
\text { (I8) }\end{array}$ \\
\hline Dificultad de acceso al edificio, casa, urbanización, etc. & $1,41 \%$ & $2,56 \%$ \\
Viviendas en las que no hay nadie & $25,68 \%$ & $46,27 \%$ \\
Viviendas en las que se niegan a recibir ninguna explicación & $5,27 \%$ & $9,23 \%$ \\
Negativas de varones a realizar la entrevista & $3,93 \%$ & $6,79 \%$ \\
Negativas de mujeres a realizar la entrevista & $4,60 \%$ & $8,57 \%$ \\
Contactos fallidos por no cumplir cuotas & $15,14 \%$ & $26,58 \%$ \\
Contactos fallidos por no ser una vivienda & & \\
(oficinas, consultas, etc.) & $43,98 \%$ & -- \\
Total personas contactadas & 21.223 & 14.179 \\
\hline
\end{tabular}

Fuente: Elaboración propia a partir de los datos del Centro de Investigaciones Sociológicas (2005a).

El trabajo de campo con encuestas telefónicas no ha sido realizado por este organismo, sino que fue subcontratado a una empresa especializada, lo que ha generado un cambio en la definición de las incidencias. En la tabla 2 se presentan las incidencias localizadas en las encuestas telefónicas distribuidas de forma "similar" a las mostradas 
en la primera Tabla. Comenzando por la parte inferior, un $26,6 \%$ de las incidencias $(8,1$ $+17,7+0,8)$ son debidas a una mala selección del marco muestral, el $11,5 \%$ están producidas por contactar con viviendas con "cuotas cubiertas" y un 22,3\% por llamadas a viviendas en las que no hay nadie.

Es importante señalar que en las encuestas telefónicas se localizan un mayor número de viviendas donde se niegan a recibir explicación (11,5\%); al tiempo que las negativas son muy superiores a las detectadas por la encuesta presencial: un $28,1 \%$. Esto se explica por la facilidad de realizar una negativa por teléfono, así como por la sencillez de cortar la comunicación (anula la entrevista en proceso). Los 10.689 contactos realizados para contactar con 1.606 personas (7.839 contactos infructuosos y 1.606 entrevistas) supone efectuar una media de 5,9 contactos por entrevistado, frente a las 10 que han sido precisas para la encuesta presencial. Esto permite concluir que "las encuestas telefónicas producen un menor número de incidencias en el trabajo de campo puesto que la facilidad de realizar rellamadas (sin coste) supone una gran reducción del número de ausencias. Téngase en cuenta que las ausencias son responsables de la mitad de las incidencias de las encuestas realizadas por el CIS entre 1996 y 2003 (Núñez 2005); tendencia que se mantiene constante en los últimos años (Díaz y Núñez 2008). En el caso de la encuesta telefónica las ausencias suponen el 22,3\% del total de incidencias (tabla 2), cifra que podría aumentar hasta el $40 \%$ si consideramos como "ausencia" la llamada a números erróneos.

Cuando se eliminan las unidades contactadas que no forman parte del marco muestral (parte derecha de la tabla 2) se observa que un $54 \%$ de las incidencias están producidas por rechazos a cooperar en la entrevista (15,7\% rechazo sin explicación y $38,3 \%$ por negativas al constatar que se trata de una encuesta), un 30\% por llamadas a teléfonos que no responden y el $16 \%$ por contactos con "cuotas cubiertas" de sexo y edad.

Finalmente, en la tabla 3 se presentan las incidencias obtenidas por la encuesta presencial y la telefónica con el fin de detectar las diferencias entre ambos procedi-mientos. En la encuesta presencial destacan los problemas de accesibilidad a la vivienda y una mayor tasa de contactos fallidos por no cumplir cuota; si bien la encuesta telefónica presenta una mayor tasa de respuesta fundamentalmente debido a la ausencia de problemas de accesibilidad y el menor número de contactos fallidos por estar cubierta la cuota 5 . Resulta sorprendente la gran cantidad de viviendas en las que no hay nadie, fundamentalmente por la facilidad que presentan los sistemas CATI para hacer rellamadas durante diferentes días y a distintas horas. De la información de la ficha de incidencias se desprende que han realizado hasta 12 llamadas durante 11 días 6 .

\footnotetext{
${ }^{5} \mathrm{O}$ al menos estos no han sido recogidos por la plantilla de incidencias. Como han señalado trabajos realizados en otros contextos, los contestadores automáticos, la identificación de número entrante, etc. son habitualmente utilizados con el fin de "filtrar llamadas". En este estudio o no se han producido o bien no han sido identificados en la ficha de incidencias utilizada.

${ }^{6}$ Entre el 26 de mayo y el 5 de junio de 2005.
} 
Tabla 2.

Incidencias en el proceso de localización del entrevistado en entrevistas telefónicas.

\begin{tabular}{|c|c|c|c|}
\hline & \multirow[t]{2}{*}{ Número } & \multicolumn{2}{|c|}{ Porcentajes } \\
\hline & & $\begin{array}{c}\text { Todas } \\
\text { las incidencias }\end{array}$ & $\begin{array}{l}\text { Sin "no } \\
\text { viviendas" }\end{array}$ \\
\hline \multicolumn{4}{|l|}{ Viviendas vacías, ausencias (I.3) } \\
\hline No descuelga el teléfono / ausencia hogar & 2.381 & $22,3 \%$ & $30,4 \%$ \\
\hline \multicolumn{4}{|l|}{ Rechazo sin explicación (I.4) } \\
\hline Cuelga inmediatamente, no da razones & 1.228 & $11,5 \%$ & $15,7 \%$ \\
\hline Negativa tras constatar que se trata de una encuesta (I.5 e I.6) & & & $38,3 \%$ \\
\hline Anula entrevista en proceso (corta a mitad de la encuesta) & 256 & $2,4 \%$ & \\
\hline No tiene tiempo, mal momento & 631 & $5,9 \%$ & \\
\hline No participa nunca en encuestas, no confía en encuestas & 70 & $0,7 \%$ & \\
\hline Negativas, no le interesa & 1.533 & $14,3 \%$ & \\
\hline No se siente capacitado para hacer encuesta & 509 & $4,8 \%$ & \\
\hline Contactos fallidos por cuotas cubiertas (I.7) & & & $15,7 \%$ \\
\hline Fuera de cuota & 1.229 & $11,5 \%$ & \\
\hline Encuestas desechadas por fuera de cuota & 2 & $0,0 \%$ & \\
\hline \multicolumn{4}{|l|}{ No viviendas (I.8) } \\
\hline Lugares de trabajo & 866 & $8,1 \%$ & \\
\hline \multicolumn{4}{|l|}{ Otros problemas con el marco muestral } \\
\hline Teléfono erróneo & 1.897 & $17,7 \%$ & \\
\hline Trabaja en el sector & 4 & $0,0 \%$ & \\
\hline Contactos sin derecho a voto & 83 & $0,8 \%$ & \\
\hline Total contactos realizados (teléfonos utilizados) & 10.689 & $100,0 \%$ & 7.839 \\
\hline
\end{tabular}

Fuente: Centro de Investigaciones Sociológicas (2005a). Información proporcionada por el CIS.

La utilización de rellamadas genera, en contrapartida, un aumento en el número de rechazos, en la medida que los "no contactos" se "convierten" en rechazos". El 54\% de las incidencias localizadas por las encuestas telefónicas corresponden a rechazos a cooperar, si bien sorprende la alta tasa de "viviendas en las que se niegan a recibir explicación" (rechazo directo), en la medida que es un rechazo producido antes de escuchar que se trata de una encuesta. El gran empleo del medio telefónico para publicidad y acciones de telemarketing puede explicar este hecho (Smith 1995:157 y Holbrook et al., 2003:82-88).

${ }^{7}$ La insistencia en el contacto genera un mayor número de rechazos. 
Tabla 3.

Comparación entre las incidencias en encuestas presenciales y telefónicas.

\begin{tabular}{llcc}
\hline & & Presencial & Telefónica \\
\hline I.2 & Dificultad de acceso al edificio, casa, urbanización, etc. & $2,56 \%$ & No procede \\
I.3 & Viviendas en las que no hay nadie & $46,27 \%$ & $30,4 \%$ \\
I.4 & Viviendas en las que se niegan a recibir ninguna explicación & $9,23 \%$ & $15,7 \%$ \\
I.5-6 & Negativas (varones y mujeres) a realizar la entrevista & $15,36 \%$ & $38,3 \%$ \\
I.7 & Contactos fallidos por no cumplir cuotas & $26,58 \%$ & $15,7 \%$ \\
& Total personas contactadas & 14.179 & 7.839 \\
& Tamaño muestral & 1.599 & 1.606 \\
\hline
\end{tabular}

Fuente: Elaboración propia a partir de los datos del Centro de Investigaciones Sociológicas (2005a).

\section{REPRESENTATIVIDAD MUESTRAL SEGÚN PROCEDIMIENTO}

Expuesto el proceso de respuesta, a continuación centraremos nuestra atención en conocer la adecuación muestral según cada procedimiento de recogida de información, buscando comprobar la segunda hipótesis que daba cuenta de la existencia de importantes diferencias muestrales entre ambos procedimientos. Para llevar a cabo el contraste utilizaremos los datos desagregados de edad y sexo del Padrón Municipal de Habitantes y la Encuesta de Población Activa del año 2005 (Instituto Nacional de Estadística 2005; 2006).

Los dos procedimientos revelan escasas desviaciones respecto al universo considerando la edad y el sexo, aunque se produce una ligera sobrerrepresentación de las personas más jóvenes y los mayores (parte superior del gráfico 1). Ambos procedimientos producen aumentos considerables a la hora de estimar la tasa de actividad y paro, si bien el análisis de cada uno por separado desvela que la encuesta presencial realiza una "mejor estimación" que la telefónica (parte superior del gráfico 1). En la encuesta presencial la diferencia en la "tasa de actividad" es de 1,6 puntos, reduciéndose a 0,3 en el caso de los hombres; mientras que en la encuesta telefónica esta diferencia llega a 2,5 puntos en la tasa de actividad total, reduciéndose a 2 en el caso de los hombres. Similares conclusiones se obtienen al considerar la "tasa de paro": la encuesta presencial la sobreestima en 5,1 puntos (reduciéndose a 1,9 en los hombres), mientras que la sobreestimación de la encuesta telefónica alcanza los 6,3 puntos). Respecto a la "tasa de empleo" la encuesta presencial realiza una mejor precisión en el caso de los hombres, mientras que la telefónica ajusta mejor al estimar la tasa de empleo femenina (gráfico 1). En definitiva, las encuestas telefónicas consiguen entrevistar un mayor número de personas "activas" (laboralmente hablando), si bien la presencial realiza una mejor representación que la telefónica. 
Gráfico 1.

Comparación entre la muestra conseguida y el universo.

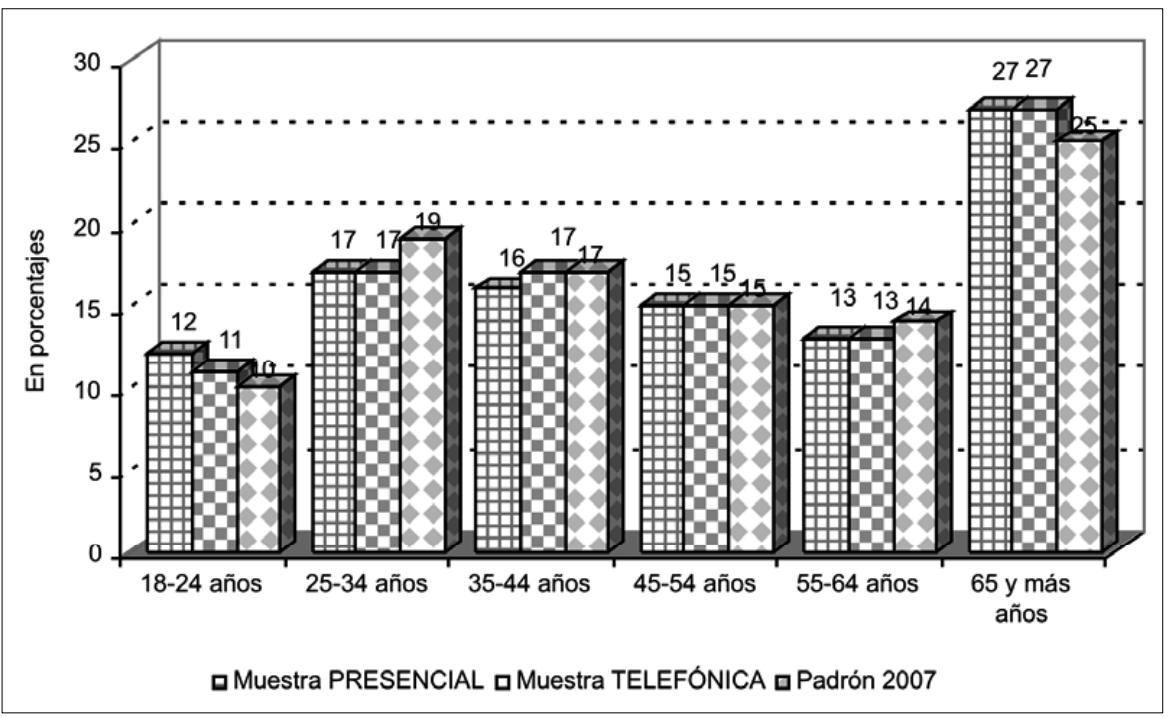

Tasa de actividad

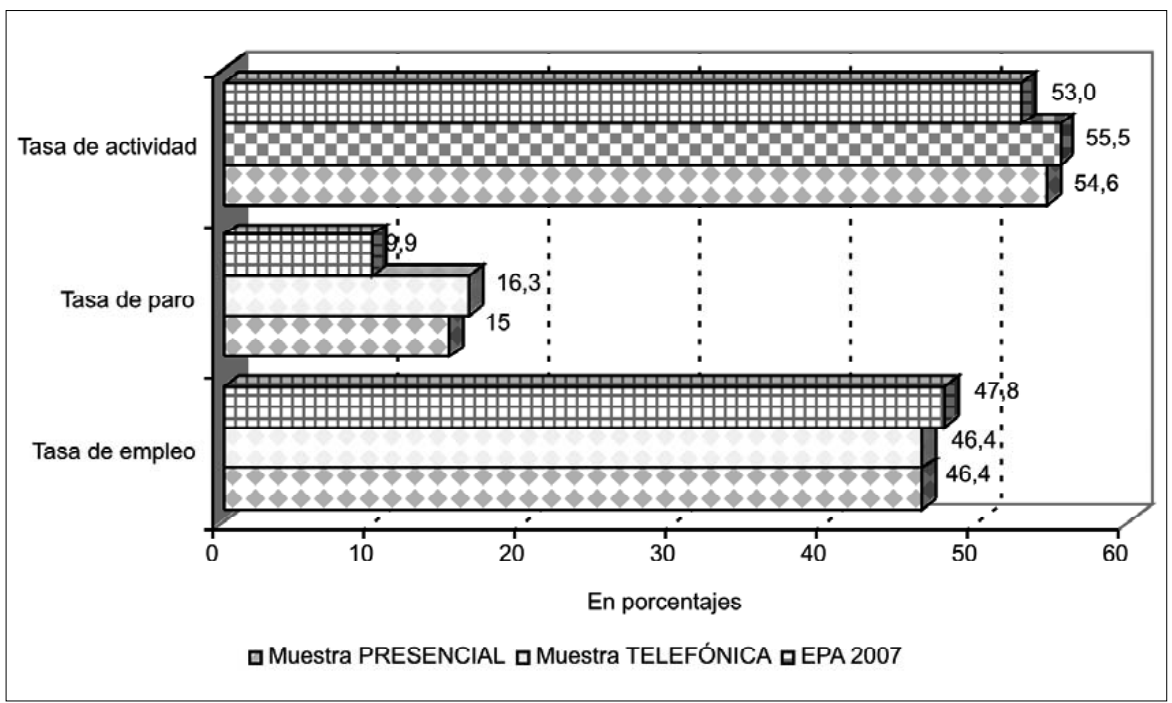

Fuente: Elaboración propia a partir de los datos del Centro de Investigaciones Sociológicas (2005a). Los datos del universo corresponden al Padrón y la EPA del año 2007. 
Al analizar estas diferencias considerando el sexo de los entrevistados revela que la encuesta presencial representa mejor la actividad de los hombres (diferencias respecto al universo de 3,1 en la encuesta presencial y de 9,9 en la telefónica); produciéndose menores diferencias en la estimación realizada por ambos procedimientos en el caso de las mujeres (13,2 y 11,8, respectivamente). De esto se deduce que las diferencias en la "representatividad total" están provocadas, fundamentalmente, por los desajustes en el caso de las mujeres.

El nivel de estudios es la variable que presenta unas mayores desviaciones respecto al universo, causadas fundamentalmente por una mala selección de los colectivos con estudios intermedios (primarios y secundarios). La encuesta personal selecciona un mayor número de personas con bajos estudios (sin estudios y estudios primarios), mientras que la encuesta telefónica lleva a cabo una mayor selección de personas con estudios medios-altos (secundarios y universitarios), en línea con la tendencia detectada por otras investigaciones (Wessell et al. 2000:4; Green et al. 2001:1; Bowers y Ensley 2003:5). Ahora bien, y en contra de los hallazgos localizados en otros contextos, la encuesta telefónica proporciona un mejor ajuste que la personal; la encuesta telefónica es la que mejor reproduce el nivel educativo de la población española.

\section{Calidad en la Respuesta (erRores de observación)}

En el apartado anterior se analizaron los errores de "no observación"; aquellos que surgen por no seleccionar correctamente una muestra de la población objeto de estudio. Siendo la tasa de respuesta una condición necesaria, no es suficiente puesto que de poco sirve una elevada tasa de respuesta si los datos recogidos presentan una baja calidad que impide un correcto análisis de la información. De modo que una vez verificada la adecuación de la muestra obtenida es el momento de considerar la calidad de la información recogida, esto es, los denominados "errores de observación" (Groves 1989:295-296). Estos tienen su origen en una inadecuación en las respuestas recogidas por el instrumento de medida, y son definidos como "la diferencia entre el valor observado y el valor 'objetivo' de una determinada unidad".

La diversidad de definiciones de calidad en la respuesta, unida a la enorme importancia de este aspecto, recomienda utilizar una definición del término que sea sencilla de operativizar y que pueda ser utilizada por otros investigadores. Tras el análisis de la literatura sobre el tema la calidad en la respuesta será definida operativamente atendiendo a dos "criterios": número de no respuestas en las preguntas del cuestionario y presencia de "deseabilidad social" en las respuestas. 


\section{Número de respuestas y no respuesta parcial}

El primer criterio de la calidad en la respuesta se centra en el número de respuestas proporcionadas por el cuestionario; considerando el número de "no respuestas" en cada una de las preguntas. Este fenómeno, conocido como "no respuesta parcial", tiene una gran importancia en la investigación mediante encuesta no sólo por la pérdida de una información (en ocasiones muy valiosa y fundamental), sino también por las implicaciones que genera en el análisis de datos; especialmente cuando se trabaja con técnicas de análisis multivariantes. Investigaciones realizadas en otros contextos (entre otros DeLeeuw 1992:55-57; Krosnick 1991; 1999) han demostrado que la encuesta presencial proporciona el menor número de preguntas no respondidas, seguida de la telefónica y las encuestas autorrellenadas (postales, vía web, etc.).

Como se muestra en la tabla 4 , un $17,6 \%$ de los entrevistados deja sin responder una pregunta en la encuesta presencial y el $13 \%$ dos. Un $46,4 \%$ de los entrevistados cara a cara $(10,2+7,8+28,4)$ dejan sin responder tres y más preguntas; porcentaje que se reduce a la mitad en la encuesta telefónica. Por otro lado, el $44 \%$ de los entrevistados a través del teléfono responden a todo el cuestionario, y uno de cada cinco $(21 \%)$ deja sin responder una pregunta. La encuesta telefónica con el sistema CATI produce una notable mayor calidad en la respuesta, sin duda por la menor complejidad que supone la realización de encuestas con el apoyo de un ordenador, presentando unos resultados diferentes a lo detectado en otros contextos. Estudios recientes realizados en el Reino Unido y en los Estados Unidos señalan que la encuesta telefónica presenta una peor cumplimentación del cuestionario (Nicolaas et al. 2000:29 y Holbrook et al. 2003:97, 104 y 107).

Los distintos componentes de la "no respuesta parcial" presentan similares conclusiones. La ausencia de respuesta puede ser provocada porque el entrevistado no responde, o bien porque "no sabe" (o no tiene opinión) sobre lo que se le pregunta. Pudiera suceder también que no se hubiera registrado ninguna respuesta en el espacio del cuestionario destinado para esa pregunta. Respecto a las respuestas "no sabe", la mitad de los entrevistados con encuestas telefónicas no proporcionan "no respuestas" de este tipo, porcentaje que aumenta al $29 \%$ en la encuesta presencial. De hecho, la mitad de los entrevistados cara a cara señalan dos y más no respuestas provocadas por el "no sabe".

La no respuesta producida por el "no contesta" presenta una tendencia similar, aunque con menos diferencias según el procedimiento utilizado: la encuesta presencial vuelve a proporcionar una menor respuesta, si bien las diferencias no son tan acusadas como en los casos anteriores. Señalamos en el párrafo anterior que un tercer componente de la no respuesta es el no registro de ninguna respuesta por el encuestador, bien porque el encuestado no ha proporcionado respuesta o porque el propio encuestador olvidó anotarla. En este estudio no se produce este efecto, ninguna pregunta aparece vacía. 
Tabla 4.

No respuesta parcial en preguntas que deben ser respondidas por toda la muestra.

\begin{tabular}{|c|c|c|c|}
\hline \multicolumn{4}{|c|}{$\begin{array}{l}\text { Considerando toda la no respuesta parcial } \\
\text { (no sabe y no contesta) }\end{array}$} \\
\hline & $\begin{array}{l}\text { Encuesta } \\
\text { presencial }\end{array}$ & $\begin{array}{l}\text { Encuesta } \\
\text { telefónica }\end{array}$ & $\begin{array}{l}\text { Toda la } \\
\text { muestra }\end{array}$ \\
\hline \multicolumn{4}{|c|}{ No respuesta parcial $\left(\mathrm{V}^{8}=0,298\right.$, sig. 0,000$)$} \\
\hline Ninguna (0 "no respuestas") & $-22,6 \%$ & $+44,4 \%$ & $33,5 \%$ \\
\hline Una & $-17,6 \%$ & $+21,0 \%$ & $19,3 \%$ \\
\hline Dos & $13,4 \%$ & $13,0 \%$ & $13,2 \%$ \\
\hline Tres & $+10,2 \%$ & $-7,3 \%$ & $8,7 \%$ \\
\hline Cuatro & $+7,8 \%$ & $-4,5 \%$ & $6,1 \%$ \\
\hline Entre cinco y veinticuatro & $+28,4 \%$ & $-9,8 \%$ & $19,1 \%$ \\
\hline Total & 1599 & 1606 & 3202 \\
\hline
\end{tabular}

(+) Residuos estandarizados corregidos (valor positivo) con un nivel de significación igual o menor a 0,01.

(-) Residuos estandarizados corregidos con valor negativo.

Fuente: Elaboración propia a partir de los datos del Centro de Investigaciones Sociológicas (2005a).

\section{Presencia de "deseabilidad social" en las respuestas}

El segundo criterio para evaluar la calidad de la respuesta está referido a la influencia del procedimiento en las respuestas de los entrevistados; esto es, que el hecho de ser entrevistado frente a un entrevistador 0 a través del teléfono genere unas respuestas distintas. Este aspecto hace referencia a la presencia de un sesgo de "deseabilidad social" en las respuestas que implica responder no lo que se piensa sino lo que está "bien visto" a los ojos del entrevistador, elegir la respuesta "socialmente deseable" a la persona que escucha. El juicio del entrevistador (pese a ser un desconocido), la desaprobación social de determinadas actitudes, etc., llevan al entrevistado a expresar respuestas "políticamente correctas" -que pueden no coincidir con su sentir-, respuestas mediatizadas por la "deseabilidad social".

En el Cuestionario preelectoral sobre las elecciones autonómicas gallegas hay varias preguntas susceptibles de estar influidas por este aspecto, de generar respuestas "socialmente bien vistas" a los ojos del entrevistador. Se trata, concretamente, de las relacionadas con la intención de votar (o abstenerse) y con la opción elegida para

\footnotetext{
${ }^{8} \mathrm{~V}$ de Cramer y significación.
} 
votar (voto político); temáticas que han sido definidas como "sensibles" por numerosos trabajos (Krosnick 1999:545-546; Belli et al. 2001; Bernstein et al. 2001; Harbaugh 1996; Hawaday et al. 1993; Jäckle et al. 2006:12; Karp y Brockington 2005; Nicolaas et al. 2000:37-38; Presser 1990; Silver et al. 1986 y Voogt et al. 2005). Nos centraremos en la segunda, la opción elegida para votar, puesto que la primera (intención de votar 0 abstenerse) ha sido expuesta en otro trabajo del autor (Díaz 2007).

La intención de voto se obtiene preguntando sobre el partido al que votaría si mañana hubiera elecciones generales. Los que no responden a un partido concreto son interrogados de nuevo por el partido hacia el que siente más simpatía, o el que se encuentra más cercano a sus propias ideas (preguntas 11 y 11a). Resultan sorprendentes las grandes diferencias entre la intención de voto según el procedimiento de administración del cuestionario (tabla 5). Dos aspectos llaman la atención. El primero es la "mejor respuesta" proporcionada por los entrevistados a través del teléfono, mejor respuesta definida como adecuación de lo que plantea la pregunta. En la encuesta telefónica el $79 \%$ ha señalado un partido concreto, frente al $68 \%$ en la presencial, hecho provocado por el elevado número de personas que declaran -en la encuesta presencialque "no saben todavía" y que no contestan la pregunta. Esta misma tendencia se detecta cuando se analizan las preguntas del cuestionario referidas a partidos políticos: concretamente al partido que le gustaría que ganara, el que cree que ganará (los que responden PP son preguntados después si creen que obtendrá mayoría absoluta), y el pacto de gobierno preferido para gobernar Galicia. En todas ellas la encuesta presencial presenta una menor respuesta, un mayor porcentaje de no sabe y no contesta.

Porcentajes de respuestas "no sabe" y "no contesta":

- Pregunta 12 (desea que gane): Encuesta presencial 24,3\%, encuesta telefónica 11,6\%. - Pregunta 13 (cree que ganará): Encuesta presencial 24,0\%, encuesta telefónica 17,2\%. - Pregunta 13a (PP mayoría absoluta): Enc. presencial 15,3\%, encuesta telefónica 6,2\%.

- Pregunta 14 (pactos): Encuesta presencial 27,8\%, encuesta telefónica 11,0\%. 
Tabla 5.

Influencia de la deseabilidad social en la intención de voto.

Preg. 11, Intención de voto:

Suponiendo que las elecciones al Parlamento Gallego se celebrasen mañana, ¿a qué partido o coalición votaría Ud.?

\begin{tabular}{lccc}
\hline & $\begin{array}{c}\text { Encuesta } \\
\text { presencial }\end{array}$ & $\begin{array}{c}\text { Encuesta } \\
\text { telefónica }\end{array}$ & $\begin{array}{r}\text { Toda la } \\
\text { muestra }\end{array}$ \\
\hline Intención de voto (V=0,174, sig. 0,000) & & & \\
$\quad$ Partido Popular (PP) & $37,2 \%$ & $32,9 \%$ & $33,1 \%$ \\
Bloque Nacionalista Galego (BNG) & $-12,9 \%$ & $+17,6 \%$ & $15,2 \%$ \\
Partido Socialista de Galicia (PsdeG), PSOE & $-21,0 \%$ & $+28,0 \%$ & $24,5 \%$ \\
EU-IU & $0,9 \%$ & $-0,8 \%$ & $0,8 \%$ \\
Otros partidos & $0,1 \%$ & $-0,1 \%$ & $0,1 \%$ \\
Suma (Voto decidido) & $68,0 \%$ & $79,3 \%$ & $73,7 \%$ \\
En blanco & $-1,9 \%$ & $+3,5 \%$ & $2,7 \%$ \\
No votaría & $+3,7 \%$ & $-2,5 \%$ & $3,1 \%$ \\
No sabe todavía & $+16,7 \%$ & $-7,9 \%$ & $12,3 \%$ \\
No contesta & $+9,6 \%$ & $-6,8 \%$ & $8,2 \%$ \\
Total & 1.599 & 1.606 & 3.305 \\
\hline
\end{tabular}

Preg. 11a: Simpatía hacia partidos

(Respondida por los que han elegido las cuatro últimas opciones en la pregunta 11) En todo caso, ¿por cuál de los siguientes partidos o coaliciones siente usted más simpatía, 0 cuál considera más cercano a sus propias ideas? (Simpatía)

\begin{tabular}{lccc}
\hline & $\begin{array}{c}\text { Encuesta } \\
\text { presencial }\end{array}$ & $\begin{array}{c}\text { Encuesta } \\
\text { telefónica }\end{array}$ & $\begin{array}{r}\text { Toda la } \\
\text { muestra }\end{array}$ \\
\hline Simpatía hacia partidos (V=0,240, sig. 0,000) & & & $17,3 \%$ \\
PP & $-13,3 \%$ & $+23,5 \%$ & $7,5 \%$ \\
BNG & $-5,3 \%$ & $+10,8 \%$ & $12,5 \%$ \\
PsdeG, PSOE & $-10,2 \%$ & $+16,0 \%$ & $0,7 \%$ \\
EU-IU & $-0,2 \%$ & $+1,5 \%$ & $38,0 \%$ \\
Suma (Voto decidido) & $29,0 \%$ & $52,0 \%$ & $26,1 \%$ \\
Ninguno & $+30,5 \%$ & $-19,3 \%$ & $13,9 \%$ \\
No sabe & $+15,1 \%$ & $-12,0 \%$ & $22,1 \%$ \\
No contesta & $+25,4 \%$ & $-16,8 \%$ & 844 \\
Total & 511 & 333 & \\
\hline
\end{tabular}

Fuente: Elaboración propia con datos del Centro de Investigaciones Sociológicas (2005a).

${ }^{9}$ Téngase en cuenta que se trata de datos; considerando el mismo número de entrevistas por cada provincia. En las tablas 6 y 7 serán presentada esta información con datos ponderados, considerando cada entrevistado con su peso proporcional dentro de cada provincia. 
Volviendo de nuevo a las respuestas de la pregunta 11 (tabla 5) el segundo aspecto que llama la atención es que la encuesta telefónica obtiene un mayor número de votantes del BNG y del PSOE. En una situación de mayoría absoluta por parte del PP10, ¿pudiera ser que las personas entrevistadas mediante entrevista presencial "ocultaran" su intención de votar a otros partidos? ¿O más bien podría hablarse de la presencia de un efecto "deseabilidad social" al declarar su voto al partido ganador de las elecciones anteriores? Investigaciones realizadas en otros contextos (Nicolaas et al. 2000:38, 46) utilizan esta argumentación para explicar una situación similar.

Aquellos que no respondieron con precisión a la pregunta del voto fueron preguntados por la "simpatía" (o cercanía) hacia partidos. De nuevo la encuesta presencial presenta una mayor número de respuestas imprecisas: un 30,5\% dice que no votará a ninguno, el $25,4 \%$ no responde, y un $15 \%$ no sabe. En total, un $71 \%$ de los entrevistados no proporcionan una respuesta definida, porcentaje que se reduce al $48 \%$ en la encuesta telefónica.

Eliminando los entrevistados que no señalan un partido concreto (esto es, las últimas categorías de la pregunta 11, ver tabla 5), y ponderando los resultados considerando la distribución proporcional de las entrevistas ${ }^{11}$, el PP es elegido por el $43,7 \%$ de los entrevistados, el PSdeG/PSOE por el $33,5 \%$ y el BNG por un $21,2 \%$. Como puede observarse en la tabla 6 se trata de resultados muy cercanos a la estimación de voto realizada por el CIS (Centro de Investigaciones Sociológicas 2005b), y también muy similares a los resultados electorales. Las desviaciones localizadas son la sobreestimación de EU/IU y PSdeG/PSOE en un $2 \%$ y $1 \%$ respectivamente, y la infraestimación del PP en un $1,2 \%$. Se trata de desviaciones que pueden ser perfectamente explicadas aludiendo al error muestral.

\footnotetext{
${ }^{10}$ En el momento de realizar el sondeo, Galicia estaba gobernada por el Partido Popular, consecuencia de conseguir en las elecciones del año 2001 el $51,6 \%$ de los votos. De los 75 diputados del Parlamento Gallego, 41 eran del PP.

${ }^{11}$ La distribución muestral se realizó mediante una "afijación uniforme" del tamaño muestral en cada provincia (800 encuestas en cada una); si bien las grandes diferencias de población en cada provincia precisan, cuando se deseen presentar resultados para toda Galicia, realizar una ponderación de los resultados que "devuelvan" a cada provincia la proporcionalidad. Hasta ahora se han presentado los datos "directos", tal y como fueron recogidos, y a partir de este momento se presentarán los datos ponderados, "devolviendo" a cada provincia su proporcionalidad correspondiente.
} 
Tabla 6.

Intención de voto (y simpatía hacia partidos) ${ }^{12}$ y estimación de voto utilizando la estimación realizada por el CIS.

\begin{tabular}{lcccc}
\hline & \multicolumn{2}{c}{$\begin{array}{c}\text { Declaración del voto en la } \\
\text { encuesta (voto + simpatía) } \\
\text { Preg. completa }\end{array}$} & $\begin{array}{c}\text { Sin abs, NS y NC } \\
\text { (en \% sobre } \\
\text { voto válido) }\end{array}$ & $\begin{array}{c}\text { Resultados } \\
\text { electorales }\end{array}$ \\
\hline PP & $36,3 \%$ & $43,7 \%$ & $43,8 \%$ & $44,9 \%$ \\
PSdeG/PSOE & $27,9 \%$ & $33,5 \%$ & $32,7 \%$ & $32,5 \%$ \\
BNG & $17,6 \%$ & $21,2 \%$ & $21,7 \%$ & $19,6 \%$ \\
El//U & $1,2 \%$ & $1,4 \%$ & $1,7 \%$ & \\
Otros partidos & $0,1 \%$ & $0,2 \%$ & $0,1 \%$ & $3,0 \%$ \\
Abstención & $7,2 \%$ & & & \\
No sabe & $3,8 \%$ & & & \\
No contesta & $5,9 \%$ & & &
\end{tabular}

Diferencias respecto a los resultados electorales

\begin{tabular}{lrrrr}
\hline & $\begin{array}{c}\text { Intención } \\
\text { de voto }\end{array}$ & Simpatía & $\begin{array}{c}\text { Voto }+ \\
\text { simpatía }\end{array}$ & $\begin{array}{c}\text { Estimación } \\
\text { CIS }\end{array}$ \\
\hline PP & $-1,6$ & 1,7 & $-1,2$ & $-1,1$ \\
PSdeG/PSOE & 1,4 & $-1,3$ & 1,0 & 0,2 \\
BNG & 1,8 & 0,3 & 1,6 & 2,1 \\
Otros partidos & $-1,6$ & $-0,7$ & $-1,4$ & $-1,2$ \\
Suma & 6,4 & 4,0 & 5,2 & 4,6 \\
\hline
\end{tabular}

Fuente: Elaboración propia, a partir de los datos del Centro de Investigaciones Sociológicas (2005a; 2005b).

Esta coincidencia en los resultados desciende notablemente cuando se considera el procedimiento de recogida de información. La comparación de cada procedimiento con la estimación realizada por toda la muestra revela que la encuesta presencial sobreestima el voto al Partido Popular en un $4 \%$, al tiempo que realiza una infraestimación en el caso del PSdeG/PSOE y BNG en un $2 \%$ (tabla 7). Las desviaciones en la encuesta telefónica operan en sentido inverso: un menor voto al PP $(3,4 \%)$ y mayor en el caso del PSdeG/ PSOE y BNG. Agregando los desajustes en la estimación -sin considerar el signo- se obtiene que la encuesta telefónica realiza una estimación ligeramente más exacta que la presencial (diferencias 6,8 y 7,9 respectivamente), si bien la diferencia es mínima.

\footnotetext{
${ }^{12}$ Las diferencias respecto a la tabla 5 tienen lugar porque aquí se trata de datos ponderados respetando la desigual población de cada provincia.
} 
Ahora bien, cuando las estimaciones realizadas por cada procedimiento se comparan con los "resultados electorales" las diferencias disminuyen en la encuesta presencial (mejor estimación), y aumentan notablemente en el caso de la encuesta telefónica. La encuesta presencial sobreestima el voto al PP en un 2,7\% e infraestima los resultados del PSdeG/PSOE en un 1\%. Los desajustes totales respecto a los resultados electorales son del $5,3 \%$. La encuesta telefónica, por su parte, produce una infraestimación del PP en $4,6 \%$, y una sobreestimación de PSdeG/PSOE y BNG en 2,8 y $3,2 \%$ respectivamente; lo que implica unos desajustes, respecto a los resultados electorales, en $13,6 \%$. Obsérvese, por último, que ambas encuestas realizan una mejor estimación (diferencia $5,2)$ que cada una por separado.

Tabla 7.

Intención de voto y simpatía hacia partidos según procedimiento de recogida de información. Comparación con los resultados electorales (porcentajes verticales).

\begin{tabular}{lcccc}
\hline \multicolumn{5}{c}{ Intención de voto y simpatía hacia partidos } \\
& $\begin{array}{c}\text { Encuesta } \\
\text { presencial }\end{array}$ & $\begin{array}{c}\text { Encuesta } \\
\text { telefónica }\end{array}$ & $\begin{array}{c}\text { Ambas } \\
\text { encuestas }\end{array}$ & $\begin{array}{l}\text { Resultados } \\
\text { electorales }\end{array}$ \\
\hline PP & $47,6 \%$ & $40,3 \%$ & $43,7 \%$ & $44,9 \%$ \\
PSdeG/PSOE & $31,5 \%$ & $35,3 \%$ & $33,5 \%$ & $32,5 \%$ \\
BNG & $19,4 \%$ & $22,8 \%$ & $21,2 \%$ & $19,6 \%$ \\
EI/IU & $1,5 \%$ & $1,4 \%$ & $1,4 \%$ & $3,0 \%$ \\
Otros partidos & $0,1 \%$ & $0,2 \%$ & $0,2 \%$ & 1.574 .269 \\
TOTAL (n) & 1.226 & 1.440 & 2.666 & \\
\hline
\end{tabular}

Diferencias respecto a la muestra total y respecto a los resultados electorales

\begin{tabular}{|c|c|c|c|}
\hline \multicolumn{2}{|c|}{$\begin{array}{c}\text { Respecto a la } \\
\text { estimación } \\
\text { con toda la muestra }\end{array}$} & \multicolumn{2}{|c|}{$\begin{array}{l}\text { Respecto a la } \\
\text { resultados electorales }\end{array}$} \\
\hline $\begin{array}{l}\text { Encuesta } \\
\text { presencial }\end{array}$ & $\begin{array}{l}\text { Encuesta } \\
\text { telefónica }\end{array}$ & $\begin{array}{l}\text { Encuesta } \\
\text { presencial }\end{array}$ & $\begin{array}{l}\text { Encuesta } \\
\text { telefónica }\end{array}$ \\
\hline 3,9 & $-3,4$ & 2,7 & $-4,6$ \\
\hline$-2,0$ & 1,8 & $-1,0$ & 2,8 \\
\hline$-1,8$ & 1,6 & $-0,2$ & 3,2 \\
\hline 0,1 & 0,0 & & \\
\hline$-0,1$ & 0,0 & $-1,4$ & 3,0 \\
\hline 7,9 & 6,8 & 5,3 & 13,6 \\
\hline
\end{tabular}

Fuente: Centro de Investigaciones Sociológicas (2005a) y Xunta de Galicia (2005). 


\section{Conclusiones}

Siguiendo el orden prefijado por las hipótesis, presentaremos sintéticamente los principales hallazgos de esta investigación centrándonos fundamentalmente en aquellos aspectos diferentes a lo mostrado en otros países, destacando las especificidades de nuestra sociedad. Además de las particularidades culturales de nuestro país, otro aspecto que puede explicar tales diferencias es la utilización de métodos para selección de los entrevistados últimos basados en rutas aleatorias y cuotas de sexo y edad.

Esto explica, por ejemplo, que la encuesta presencial haya necesitado un mayor número de contactos que la telefónica, fundamentalmente por el menor número de incidencias detectadas por esta última ${ }^{13}$. La tasa de rechazo es superior en la encuesta telefónica (hipótesis H1), tal y como era de esperar, si bien resulta sorprendente que ésta presente un mayor número de ausencias que la presencial (30\% y $26 \%$ respectivamente) pese a la facilidad para realizar rellamadas.

Tal y como proponía la segunda hipótesis existen importantes diferencias muestrales entre ambos procedimientos puesto que la encuesta telefónica selecciona un mayor número de activos, ocupados y parados, si bien la encuesta presencial realiza una mejor estimación. La encuesta telefónica selecciona también un mayor número de personas con altos niveles de estudios y con mayor práctica religiosa.

Considerando la calidad de la respuesta (tercera hipótesis), la encuesta telefónica presenta un menor número de "no respuesta parcial", tanto si se considera el 'no sabe' como el 'no contesta', un menor número de "respuestas evasivas", y es menos sensible a los efectos de deseabilidad social. Como se ha señalado la encuesta presencial presenta unas respuestas menos extremas y una menor influencia del orden de colocación de las categorías de respuesta.

Respecto al ámbito específico de la predicción política, fin último para el que se llevo a cabo la investigación utilizada, las encuestas telefónicas producen una mayor respuesta en la intención del voto futuro. La agregación de las respuestas de ambos procedimientos estima con precisión el voto obtenido en las elecciones, si bien cuando se considera cada uno de forma independiente la encuesta presencial realiza un mejor ajuste que la telefónica.

Los resultados expuestos nos "obligan" -en las últimas líneas de este trabajo- a elegir uno u otro procedimiento con el fin de conseguir la mejor forma de conocer la realidad social; en este caso la predicción electoral de las elecciones Gallegas en el año 2005. El análisis de la tabla 6 ha desvelado la gran "similitud" entre los resultados electorales y la prospección realizada por ambas encuestas ${ }^{14}$, dando cuenta de la gran

\footnotetext{
${ }^{13}$ La encuesta presencial precisa de 9 contactos para realizar una encuesta, cifra que desciende a 6,6 en la telefónica.

${ }^{14}$ Esto es, sin considerar la estimación realizada por el Centro de Investigaciones Sociológicas basada en sus modelos.
} 
capacidad de predicción del instrumento. Ahora bien, se trata de una "buena noticia" que se veía ligeramente "empañada" cuando, en el Tabla 7, se consideró por separado la "capacidad predictiva" de cada procedimiento, con diferencias que oscilan entre 5,3 puntos (diferencia entre la encuesta presencial y los resultados electorales) y los 13,6 puntos cuando se compara la encuesta telefónica con los resultados electorales.

Esta información nos llevaría a concluir que la encuesta presencial produce una mejor estimación, conclusión que es matizada cuando, repasando todo el trabajo, se recuerda que ésta precisó un mayor número de contactos para efectuar una entrevista (tabla 3), presenta una mejor calidad en la recogida de información, esto es, que tiene un mayor número de "no respuestas" (tabla 4), y obtiene un menor número de respuestas precisas en la intención de voto (tabla 5).

Esta situación, y las diferencias en la correcta representatividad del universo objeto de estudio nos lleva a preguntarnos si realmente es preciso optar por uno u otro procedimiento y ¿por qué no utilizar ambos? Esto implicaría unir la rapidez y la economía de la encuesta telefónica con la mejor representatividad que consigue la encuesta presencial; consiguiendo así un mejor ajuste a la realidad objeto de estudio con un menor coste económico ${ }^{15}$. Considerando que cada procedimiento consigue una mayor capacidad acceso en cada colectivo (esto es, un "acceso selectivo" según la población objeto de estudio), podría ser conveniente la utilización de otros procedimientos menos costosos (por ejemplo, encuestas vía web) con el fin de recoger mejor las características de la población objeto de estudio (DeLeeuw 2005; DeLeeuw, Hox y Dillman 2008).

Esta mejora de la herramienta de la encuesta para acceder a "población difícil" debe ser combinada con cambios en el diseño del cuestionario, con el fin de adaptarlo a las características del procedimiento de recogida de información y a la población que cooperará con cada procedimiento (De Leeuw y Hox 2008). No es posible utilizar un mismo cuestionario con todos los procedimientos por el gran cambio que se produce en el proceso de interacción con el encuestado cuando se encuentra "cara a cara" con el entrevistador o cuando es "interrogado" a través del teléfono (o incluso cuando no hay encuestador en el caso de las encuestas vía web). Será necesario, además, verificar que ambos cuestionarios son equivalentes, que los cambios del cuestionario con el fin de adaptarlos a cada procedimiento no generan cambios en las respuestas. Sólo de esta forma será posible alejar al "fantasma de la no respuesta" (Brehm 1993; Singer 2006), algo que está poniendo en "verdaderos aprietos" la representatividad y el futuro de la encuesta como herramienta de investigación para el conocimiento de la realidad social (Alvira 2006).

\footnotetext{
${ }^{15}$ En la medida que resulta más económico realizar una investigación con 1.600 encuestas presenciales y 1600 telefónicas que 3200 entrevistas presenciales.
} 


\section{REFERENCIAS BIBLIOGRÁFICAS}

Alvira, F. 2006. "El futuro de la encuesta". Presentado en el IV Congreso de Metodología de Encuestas (Conferencia de apertura), 20 de septiembre, Pamplona.

Belli, R. F., M. W. Traugott y M. N. Beckman. 2001. "What leads to voting overreports? Contrast of overreporters to validated voters and admitted nonvoters in the American national election studies." Journal of Official Statistics 17:479-498.

Bernstein, R., A. Chadha y R. Montjoy. 2001. "Overreporting Voting. Why it Happens and Why It Matters." Public Opinion Quarterly 65:22-44.

Bowers, J. y M. J. Ensley. 2003. Issues in Analyzing Data from the Dual-Mode 2000 American National Election Study, NES Technical Report.

Brehm, J. 1993. The Phantom Respondents. Ann Arbor: Universidad de Michigan.

Centro de Investigaciones Sociológicas 2005a. Preelectoral de Galicia. Elecciones autonómicas 2005. Estudio n. ${ }^{\circ}$ 2608. Madrid: Centro de Investigaciones Sociológicas.

Centro de Investigaciones Sociológicas 2005b. Estimación de voto del estudio 2608/0: "Preelectoral de Galicia. Elecciones Autonómicas 2005". Consultado el 4 de octubre de 2007, (http://www.cis.es/cis/ opencms/-Archivos/Marginales/2600_2619/2608/e260800.html).

DeLeeuw, E. D. 1992. Data Quality in Mail, Telephone and Face to Face Surveys. Amsterdam: TT-Publikaties.

De Leeuw, E. 2005. "To mix or not to mix data collection modes in surveys." Journal of Official Statistics 21:233-255.

De Leeuw, E. 2008a. "Choosing the method of data collection." Pp. 113-135 en International Handbook of Survey Methodology, edited by E. D. de Leeuw, J. J. Hox y D. A. Dillman. Nueva York: Lawrence Erlbaum Associates y Asociación Europea de Metodología.

De Leeuw, E. and J. J. Hox. 2008. "Self-administered questionnaires: mail surveys and other applications." Pp. 239-264 en International Handbook of Survey Methodology, edited by E. D. de Leeuw, J. J. Hox y D. A. Dillman. Nueva York: Lawrence Erlbaum Associates y Asociación Europea de Metodología.

De Leeuw, E., J. J. Hox y D. A. Dillman. 2008. "Mixed mode surveys: when and why Pp. 299-315 en International Handbook of Survey Methodology, edited by E. D. de Leeuw, J. J. Hox y D. A. Dillman. Nueva York: Lawrence Erlbaum Associates y Asociación Europea de Metodología.

Díaz de Rada, V. 2006. "Presentación: las incidencias en la investigación con encuestas." Metodología de Encuestas. Número monográfico sobre incidencias en el trabajo de campo 2:3-10.

Díaz de Rada, V. y A. Núñez Villuendas. 2008. Estudio de las incidencias en la investigación con encuesta. El caso de los barómetros del CIS. Madrid: CIS.

Díaz de Rada, V. 2007. Comparación entre los resultados proporcionados por la encuesta telefónica y la encuesta personal: el caso de un estudio electoral (sin publicar). 
Ellis, C. y J. A. Krosnick. 1999. Comparing telephone and face to face surveys in terms of sample representativeness: a Meta-Analysis of Demographics Characteristics. Ann Arbor: Universidad de Michigan, NES (National Election Studies) Technical Reports. Consultado el 1 de marzo de 2005 (www.umich.edu/ nes/resources/papers/papers.htm).

Fowler, F. J. 2002. Survey research methods. Newbury Park, CA: Sage, Applied Social Reseach Methdods Series 1.

Gwartney, Patricia A. 2007. The Telephone Interviewer's Handbook: How to conduct standardized conversations. San Francisco: Jossey-Bass.

Green, M. C., J. A. Krosnick y A. L. Holbrook. 2001. "The survey response process in telephone and face-toface surveys. Differences in respondent satisficing and social desirability response bias". Consultado el 23 de junio de 2004 (www.umich.edu/ nes/resources/techrpts/tech-abs/tech-ab62.htm).

Groves, R. M. 1989. Survey Error and Survey Cost. Nueva York: Wiley.

Harbaugh, W. T. 1996. "If people vote because they like to, then why do so many of them lie?." Public Choice 89:63-76.

Hawaday, C. K., P. L. Marler y M. Chaves. 1993. "What the polls don't know show: A closer look at US church attendance." American Sociological Review 6:741-752.

Holbrook, A. L., M. C. Green y J. A. Krosnick. 2003. "Telephone versus face-to-face interviewing of national probability samples with long questionnaires." Public Opinion Quarterly 67:79-125.

Jäckle, A., C. Roberts y P. Lynn. 2006. "Telephone versus face-to-face interviewing: mode effects on data quality and likely causes. Report on Phase II of the ESS-Gallup Mixed Mode Methodology project." ISER Working Paper 2006-41. University of Essex, Colchester.

Karp, J. A. y D. Brockington. 2005. "Social desirability and response validity: a comparative analysis of overreporting voter turnout in five countries." The Journal of Politics 3:825-840.

Keeter, S. et al. 2000. "Consequences of reducing nonresponse in a national telephone survey." Public Opinion Quarterly 2:125-148.

Krosnick, J. A. 1991. "Response strategies for coping with the cognitive demands of attitude measures in surveys." Applied Cognitive Psychology 5:213-236.

Krosnick, J. A. 1999. "Survey research." Annual Review of Psychology 50:537-567.

Martínez de Luna, I. 2008. "Encuestas de opinión: de la teoría a la práctica." Metodología de encuestas 10:7-26.

Méndez Lago, M. y R. Martínez Casinello. 2007. "Encuestas telefónicas vs. presenciales: análisis de la encuesta pre-electoral del CIS de las elecciones gallegas de 2005." Presentado en el XV Seminario de Investigaciones Políticas y Sociológicas, 15-16 noviembre 2007, Madrid. Organizado por la Asociación Española de Estudios de Mercado, Marketing y Opinión (Aedemo).

Instituto Nacional de Estadística (INE). 2005. Padrón municipal: cifras oficiales de población a 1 de enero de 2005. Madrid: INE (www.ine.es). 
Instituto Nacional de Estadística (INE). 2006. Encuesta de Población Activa año 2005. Madrid: INE (www. ine.es).

Instituto Nacional de Estadística INE. 2010. Encuesta de Tecnologías de la Información en los Hogares, año 2009. Madrid: INE (www.ine.es).

Nicolaas, G., K. Thomson y P. Lynn. 2000. The Feasibility of Conducting Electoral Surveys in the UK by Telephone. Londres: National Centre for Social Research.

Núñez Villuendas, A. 2005. "Incidencias de la entrevista personal en la investigación por encuesta." Revista Española de Investigaciones Sociológicas 109:219-236.

Presser, S. 1990. "Can context change reduce vote overreporting?" Public Opinion Quarterly 54:586593.

Silver, B. D., B. A. Anderson and P. R. Abramson. 1986. "Who overreports voting?" American Political Science Review 2:613-624.

Singer, E. 2006. "Introduction: nonresponse Bias in Household Survey." Public Opinion Quarterly. Special Issue: Nonresponse Bias in Household Survey 5:637-345.

Smith, T. W. 1995. "Trends in Non-Response Rates." International Journal of Public Opinion Research 7:157-171.

Voogt, R. J. J. y W. E. Saris. 2005. "Mixed Mode Designs: Finding the Balance Between Nonresponse Bias and Mode Effects." Journal of Official Statistics 3:367-387.

Wessell, D., W. Rahn y T. Rudolph. 2000. An analysis of the 1998 NES Mixed-Mode Design. Ann Arbor: Universidad de Michigan: NES (National Election Studies) Technical Reports. Access 1 march 2005, (www.umich.edu/ nes/resources/papers/papers.htm).

VIDAL DÍAZ DE RADA es Licenciado y Doctor en Sociología, Licenciado en Investigación y Técnicas de Mercado, y profesor de Sociología en la Universidad Pública de Navarra, Departamento de Sociología. Es autor de diversos trabajos sobre metodología, sociología del consumo y comportamiento del consumidor; destacando entre sus últimas publicaciones "Manual del trabajo de Campo en la Encuesta" (2005); "Algunos problemas de la encuesta telefónica para la proyección electoral" (2007), y "Estudio de las incidencias en la investigación mediante encuesta: el caso de los barómetros del CIS" (Con Núñez Villendas, 2007).

RECIBIDO: $10 / 02 / 2009$

ACEPTADO: 11/01/2010

Publicado on-line: 15/04/2011 


\section{AneXo}

Ficha técnica de la investigación: Estudio CIS n. ${ }^{\circ}$ 2608: Prelectoral de Galicia. Elecciones autonómicas 2005.

Ámbito: Comunidad autónoma de Galicia.

Universo: Población de ambos sexos de 18 años o más con capacidad de voto en las elecciones autonómicas gallegas.

Tamaño de la muestra:

Diseñada: 3.200 entrevistas distribuidas uniformemente en las cuatro provincias de la comunidad autónoma (800 en cada una de ellas).

Realizada: 3.205 entrevistas.

Afijación: No proporcional.

Ponderación: Para tratar la muestra en su conjunto es necesario aplicar los siguientes coeficientes de ponderación: A Coruña 1,637; Lugo 0,537; Ourense 0,501 y Pontevedra 1,325

Puntos de muestreo: 83 municipios, con la siguiente distribución provincial:

$\begin{array}{ll}\text { A Coruña: } & 19 \\ \text { Lugo: } & 23 \\ \text { Ourense: } & 23 \\ \text { Pontevedra: } & 18\end{array}$

Procedimiento de muestreo: Polietápico, estratificado por conglomerados, con selección de las unidades primarias de muestreo (municipios) y de los hogares de forma aleatoria y de las unidades últimas (individuos) por cuotas de sexo y edad.

Los estratos se han formado por el cruce de la provincia con el tamaño del hábitat, dividido en 5 categorías: menos o igual a 2.000 habitantes; de 2.001 a 10.000; de 10.001 a 50.000; de 50.001 a 100.000, y de más de 100.000 .

Metodología de recogida de la información: Mixta. El procedimiento de recogida de la información se ha dividido en dos modalidades, de forma que se han realizado 1.606 entrevistas telefónicas, con sistema CATI, en hogares seleccionados aleatoriamente a partir de la base de datos de teléfonos de los municipios y 1.599 entrevistas personales, en hogares seleccionados por medio de rutas aleatorias.

Error muestral: Para un nivel de confianza del $95,5 \%$ (dos sigmas), y $P=Q$, el error real es de $\pm 1,8 \%$ para el conjunto de la muestra y del $\pm 3,5 \%$ para cada una de las provincias.

Fecha de realización: Del 26 de mayo al 5 de junio de 2005. 